\title{
METODOLOGÍA DE LOS PROYECTOS DE INVESTIGACIÓN*
}

Juan Camilo Aponte. MD, MMSc **

\section{Resumen}

El propósito fundamental del presente artículo es brindar una breve descripción de las fases fundamentales que deben tenerse en cuenta a lo largo del desarrollo de un proyecto de investigación en salud y recordar cuales son los requisitos que en la actualidad se tienen dentro de la división de investigaciones (DI) de la Fundación Universitaria de Ciencias de la Salud (FUCS), para poder llevar en forma adecuada esta clase de proyectos (una de las modalidades existentes dentro de los proyectos de grado ${ }^{1}$ de los estudiantes de esta institución).

Palabras clave: proyecto de investigación, trabajo de grado.

Abreviaturas: DI, división de investigaciones; FUCS, Fundación Universitaria de Ciencias de la Salud.

\section{Las fases de la investigación}

Todo proyecto de investigación en salud debe seguir de una manera rigurosa y metódica una serie de fases que garanticen el adecuado desarrollo y calidad del producto final. Las que conforman dichos proyectos de investigación son: concepción, planeación, aprobación, implementación, análisis y comunicación ${ }^{2}$ (Figura 1). La primera, de concepción, es en la cual se delimita el problema de estudio, identificando el vacío de conocimiento existente a medida que se exploran los elementos fundamentales para determinar su magnitud, importancia e impacto de su solución; una vez delimitado el problema podemos identificarlo planteando una pregunta de investigación, definiendo el objetivo general y los objetivos específicos y proponiendo la hipótesis a probar, en caso de ser necesario. Como paso final de esta primera etapa debemos realizar una evaluación de la viabilidad de nuestra pregunta, para determinar si vale la pena o no dedicar tiempo para el desarrollo de un protocolo que plantee como

Fecha recibido: 24 de abril de 2006

Fecha aceptado: 2 de mayo de 2006

* Para trabajos de grado en la Fundación Universitaria de Ciencias de la Salud.

** Coordinador de Postgrados División de Investigaciones, Instructor Asistente, Fundación Universitaria de Ciencias de la Salud. resolver nuestra duda, siguiendo los lineamientos que para ello se han establecido y se consideran los pilares fundamentales para la generación de las buenas preguntas de investigación ${ }^{3,4}$ (interesante, relevante, pertinente, novedosa, específica, medible y ética). Una vez determinamos la viabilidad de nuestra pregunta pasamos a la siguiente fase, la de planeación, en la cual desarrollamos el protocolo de investigación elaborando la carta de navegación de nuestro proyecto, la cual garantiza el cubrimiento de todos los aspectos fundamentales y relevantes para poder llevar a cabo nuestro estudio y obtener resultados de adecuada calidad. Luego de terminar la elaboración del protocolo, se debe continuar con la fase más importante para la correcta realización de cualquier proyecto, la de aprobación. En ella debemos realizar, inicialmente los investigadores y después el comité de investigación y ética del lugar donde vayamos a llevar a cabo el trabajo, el análisis de la factibilidad del proyecto que estamos proponiendo, y en caso de necesitar recursos económicos debemos contar con el aval de un ente financiador. Para iniciar la cuarta fase, la de implementación, es imprescindible contar con todas las aprobaciones de rigor de acuerdo con las necesidades del proyecto que estamos planteando (aparte de las ya mencionadas, si necesitamos permisos de algún ente local, distrital o nacional, estos se deben obtener de igual manera). En esta fase realizamos, en caso de ser necesario, 
una prueba piloto de nuestro estudio, refinamos el instrumento de medición y por último, procedemos a la recolección de los datos pertinentes. Podemos pasar entonces a la fase de análisis, en la cual, luego de haber recolectado de manera parcial o en su totalidad las variables de importancia, comenzaremos a realizar los resúmenes de información de nuestro proyecto y las pruebas estadísticas que planteamos a priori en el protocolo. Por último, una vez terminado el análisis de los datos elaboramos las diferentes comunicaciones para socializar los resultados relevantes del proyecto (el artículo de investigación y los informes final y financiero en caso de que sea necesario).

\section{Los requisitos en la FUCS}

En la FUCS se deben cumplir una serie de requisitos para poder progresar en las fases de un proyecto investigación en salud y obtener una de las aprobaciones de rigor (la de la DI) para optar un título universitario específico.

En la fase de concepción o delimitación de la pregunta, se debe contar con la aprobación de los integrantes del servicio del cual los investigadores hacen parte (en caso de enfermería el aval lo da directamente el comité de investigaciones de la facultad) mediante una presentación formal ante el servicio, el cual evalúa la viabilidad del proyecto y aprueba, mediante una comunicación del jefe del servicio, la estructuración de un protocolo que permita dar solución al problema planteado. El documento de esta primera fase lo hemos denominado en la DI Propuesta de Investigación, el cual debe contener todos y cada uno de los elementos necesarios para poder evaluar la viabilidad de una pregunta de investigación. ${ }^{5}$ Este documento debe ser elaborado por el grupo investigador en cabeza del asesor temático. El proceso se cristaliza entonces en tres documentos principales: la propuesta de investigación, la aprobación mediante comunicación del jefe del servicio de la viabilidad de la pregunta investigación y la carta de compromiso del asesor temático, en la cual se fijan los lineamientos, responsabilidades e integrantes del grupo investigador. Es importante mencionar en este punto, que en la actualidad se están conformando en la FUCS una serie de grupos y líneas de investigación, cuyo objetivo primordial es servir de guía en la definición de los intereses de investigación en las facultades de la FUCS, convirtiéndose así, en uno de los pilares en la determinación de la viabilidad de un proyecto.

Luego de haber cumplido estos requisitos, la propuesta puede evolucionar a un protocolo al cursar con la fase de planeación. Durante esta, el grupo (incluyendo por supuesto al asesor temático) junto con el asesor metodológico, se toma la tarea de desarrollar a profundidad el protocolo de investigación de acuerdo con los lineamientos definidos en la DI. ${ }^{6}$ En la primera reunión es imprescindible establecer mediante un acuerdo de todos los integrantes del grupo investigador y los asesores, las normas y responsabilidades de cada integrante para reclamar autoría al finalizar el proyecto. En la actualidad la DI se encuentra en el desarrollo de unas políticas claras para definir la autoría de los trabajos de investigación que se lleven a cabo mediante la tutoría de ésta.

Una vez se ha terminado de estructurar el protocolo, pasa la fase de aprobación (Figura 2), primero por el comité de investigaciones de cada facultad (Figura 3), ante el cual se sustenta el protocolo para definir la factibilidad del desarrollo de la investigación y determina la necesidad de que el protocolo sea evaluado por el comité de ética hospitalaria. En caso de necesitar recursos económicos, el proyecto debe ser evaluado por la entidad financiadora que el grupo investigador escoja (interno o externo) para obtener el apoyo requerido. Con el fin de tener estas aprobaciones se debe contar con el protocolo de investigación definitivo y los investigadores deben verificar que en cada uno los comités en que se presenten los proyectos, haya constancia de su aprobación en el acta. En el caso de cursar en una entidad financiadora, el grupo debe suministrar la carta de aprobación presupuestal expedida por dicha entidad. Para finalizar esta fase, el protocolo de investigación con cada una de las cartas de aprobación requeridas debe ser radicado ante la DI. 
Luego de obtener las aprobaciones de rigor se puede seguir con la implementación y conducción del estudio. Durante el transcurso de esta fase se podrán realizar auditorias por parte de la DI para verificar la correcta conducción y administración del proyecto. Además, en el momento que se cuente con la recolección total de datos, el bioestadístico de la división realizará un análisis exploratorio para verificar la calidad de los datos. Al terminar la recolección se hará una carta de certificación de la veracidad de los datos por parte del grupo investigador, y se debe contar con las aprobaciones de la implementación de la DI y del análisis exploratorio por parte del bioestadístico.

Por último, podemos analizar los datos luego de lo cual entraremos a realizar los informes requeridos en la fase de comunicación, para la finalización del proyecto, que en el caso de la FUCS es un artículo de investigación estructurado de acuerdo con las normas Vancouver ${ }^{7}$ que debe contar con las aprobaciones de los asesores metodológico y temático, el jefe del servicio y la totalidad del grupo investigador.

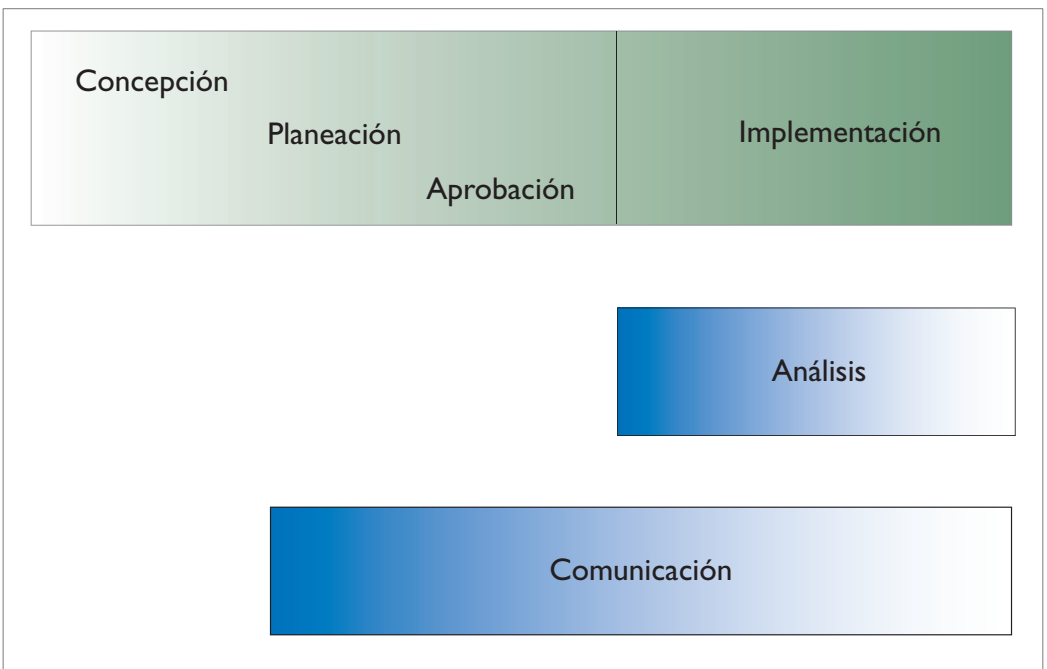

Figura I. Fases de un proyecto de investigación..

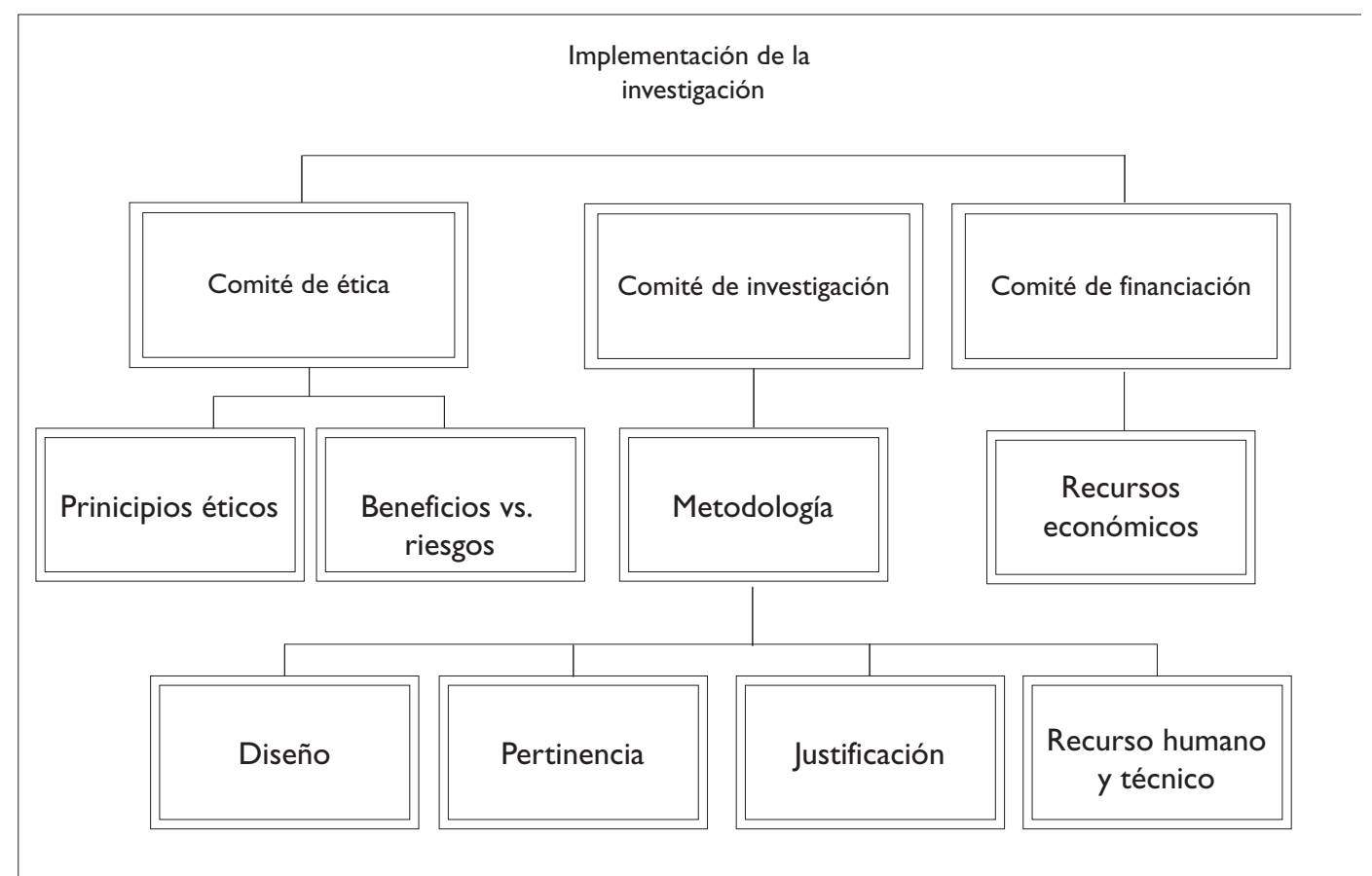

Figura 2. Definición de factibilidad del proyecto. 


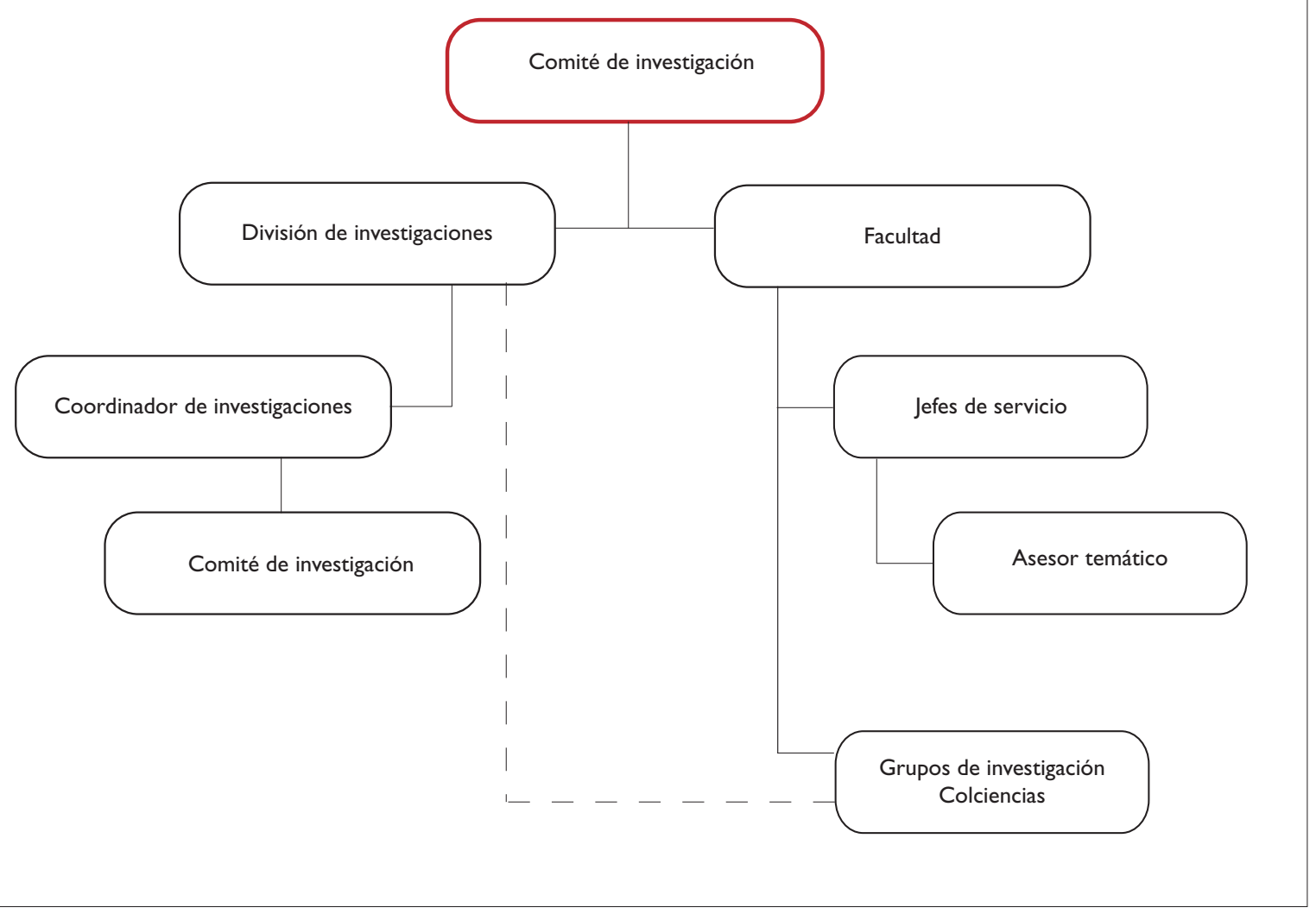

Figura 3. Comité de investigaciones.

\section{Referencias}

1. Fundación Universitaria de Ciencias de la Salud. Reglamento Estudiantil Pregrado y Postgrado. Bogotá: Fundación Universitaria de Ciencias de la Salud; 2006.

2. Lozano León JM. Investigación en salud: qué es y para qué sirve. En: Morales Ruiz Á, Morillo Zárate LE, editores. Epidemiología Clínica. Bogotá: Editorial Medica Panamericana; 2004. p. 19-28.

3. Hernández Sampieri R, Fernández Collado C, Baptista Lucio P. Planteamiento del problema: Objetivos, pregunta de investigación y justificación del estudio. En: Hernández Sampieri R, Fernández Collado C, Baptista Lucio P, editores. Metodología de la Investigación. $3^{\mathrm{a}}$. ed. México: McGrawHill Interamericana; 2006. p. 41-60.
4. Ruiz Peláez JG. La Pregunta de Investigación. In: Morales Ruiz A. Morillo Zárate LE, editors, Epidemiologìa Clínica. Bogotà: Editorial Médica Panamericana; 2004. p. $29-50$.

5. Aponte JC. Guía Para la Elaboración de una propuesta de investigación. División de Investigaciones. Editor 6-4-2006. Fundación Universitaria de Ciencias de la Salud.

6. Aponte JC. Guía Para la Elaboración de un protocolo de investigación. División de Investigaciones, editor. 6-42006. Fundación Universitaria de Ciencias de la Salud.

7. Aponte JC. Guía Para la presentación de un artículo de investigación. División de Investigaciones, editor 6-42006. Fundación Universitaria de Ciencias de la Salud.

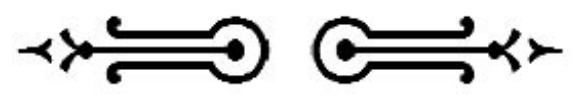

